Agnieszka Mlicka ${ }^{1}$, Tomasz Aleksiewicz ${ }^{1}$, Maciej Rydzkowski ${ }^{1}$, Paweł Niewiadomski ${ }^{1}$, Wioletta Zielińska², Marta Hałas-Wiśniewska², Klaudia Mikołajczyk², Magdalena Izdebska1, 2

${ }^{1}$ Students research group of Cell Biology and Ultrastructure at Department of Histology and Embryology, Nicolaus Copernicus University in Torun, Collegium Medicum in Bydgoszcz, Poland

2Department of Histology and Embryology, Nicolaus Copernicus University in Toruń, Faculty of Medicine, Collegium Medicum in Bydgoszcz, Poland

\title{
Influence of dexamethasone and doxorubicin on inhibition of hypoxia- -induced metastatic potential in HepG2 cell line
}

\section{Corresponding author:}

Magdalena Izdebska, Department of Histology and Embryology, Nicolaus Copernicus University in Torun, Collegium Medicum in Bydgoszcz, 24 Karłowicza St., 85-092 Bydgoszcz, Poland, e-mail: mizdebska@cm.umk.pl

Medical Research Journal 2021; Volume 6, Number 3, 194-203 10.5603/MRJ.a2021.0036 Copyright (C) 2021 Via Medica ISSN 2451-2591 e-ISSN 2451-4101

\begin{abstract}
One of the commonly applied methods in the case of median to advance stages of liver cancer is the transarterial chemoembolization (TACE) procedure. It involves the administration of relatively high doses of cytostatics to the tumour-supplying artery followed by the embolization of the vessel. It limits the drug action almost only to the tumour mass. However, this also reduces the availability of oxygen, which stimulates cell migration. Therefore, the study aimed to assess how the introduction of an additional drug - dexamethasone and its combination with doxorubicin will impact the viability and migration of HepG2 cells under hypoxia-mimic conditions. To assess the basic response of the cells to the drugs and evaluate the interaction between them MTT assay and apoptosis assay were used. To analyse the migratory potential transwell migration assay was applied. Epithelial-mesenchymal transition (EMT) markers and apoptosis-related proteins were studied using Western blot assay. Hypoxia-mimic conditions were induced using pretreatment with cobalt chloride. The obtained results suggest that the developed doxorubicin: dexamethasone combination limits hypoxia-induced increase in the migratory potential of HCC cells, which is connected with the inhibition of the EMT process and directing cells to death on the cellular level. Key words: hepatocellular carcinoma, doxorubicin, dexamethasone, metastasis, hypoxia
\end{abstract}

Med Res J 2021; 6 (3): 194-203

\section{Introduction}

Hepatocellular carcinoma (HCC) is in fifth place among the most common cancers in the world. The most effective method of $\mathrm{HCC}$ treatment is radical surgery, however, it is rarely possible. Another therapeutic option is transarterial chemoembolization (TACE) [1]. The procedure consists of administering cytostatics directly to the tumour-supplying artery and embolization of the vessel. This limits blood flow to the tumour, thereby reducing the oxygen level inside the tumour mass. Moreover, the direct delivery of the drug results in an enhanced anti-tumour effect and simultaneously reduces adverse reactions [2]. The most widely used cytostatic for TACE in HCC is anthracycline doxorubicin
(DOX) [3]. The use of DOX in TACE is generally accepted, although drug resistance is often seen in HCC [4]. A significant cause of chemoresistance is hypoxia. This is especially important considering the TACE procedure, which results in the induction of acute hypoxia [5]. Many reports indicate that the heterodimer of the hypoxia-inducible factor $1 \alpha$ (HIF-1 $\alpha$ ) signalling pathway induces epithelial-mesenchymal transition (EMT) in cancer cells [6]. During the EMT process, tumour cells lose epithelial cell traits and acquire mesenchymal characteristics, which favours growth, invasion, and metastasis of cancer cells [7].

Considering the huge impact of HIF- $1 \alpha$ on drug resistance and cancer development, compounds able to affect the protein are searched. One of the chemical 
compounds with such properties is dexamethasone (DEX). Many reports indicate that the level of HIF-1 $\alpha$ was decreased after the treatment of DEX in colorectal and breast cancer cells [8].

Hence the study aimed to indicate the possibility of reducing the EMT process by using combination therapy composed of DOX and DEX in hypoxia-mimic conditions in the HepG2 cell line.

\section{Materials and methods}

\section{Cell culture and treatment}

HepG2 cell line was obtained from the Department of Histology and Embryology, Medical University in Wrocław, Poland. The cells were grown in EMEM (LONZA, Basel, Switzerland) with the addition of $10 \%$ (v/v) fetal bovine serum (FBS, Sigma-Aldrich, Merck KGaA, Darmstadt, Germany) and $50 \mu \mathrm{g} / \mathrm{mL}$ gentamycin (Sigma-Aldrich) in standard culture conditions. For the cell viability analysis cells were treated with 3,4 , 5, 6, $7 \mu \mathrm{M}$ DOX (Sigma-Aldrich); 3, 4, 5, 6, $7 \mu \mathrm{M}$ DEX (Sigma-Aldrich) or the combination of DOX and DEX in the $1: 1$ ratio for $24 \mathrm{~h}$. Control cells were grown under identical conditions but in the absence of DOX and DEX. Based on the MTT assay, for the other experiments, 3, $6 \mu \mathrm{M} \mathrm{DOX;} \mathrm{3,} 6 \mu \mathrm{M}$ DEX and the combination 3:3 $\mu \mathrm{M}$ DOX/DEX and 6:6 $\mu \mathrm{M}$ DEX were used. The hypoxic microenvironment was mimicked using cobalt chloride $\left(\mathrm{CoCl}_{2}\right.$, Sigma-Aldrich). To assess whether $\mathrm{CoCl}_{2}$ impacts cell viability and HIF-1 level, the cells were incubated with $60,80,100,120,140 \mu \mathrm{M}$ concentrations of $\mathrm{CoCl}_{2}$. Based on the obtained results $80 \mu \mathrm{M}$ concentration was selected to induce hypoxia during further experiments.

\section{MTT assay and interaction between the drugs}

To determine the cytotoxic effect of DOX, DEX, and their combination the colourimetric MTT assay was used. After $24 \mathrm{~h}$, cells were washed with PBS and incubated with MTT working solution for $3 \mathrm{~h}$ in standard culture conditions. The formazan crystals were dissolved in $2 \mathrm{~mL}$ isopropanol $\left(10 \mathrm{~min}, 37^{\circ} \mathrm{C}\right.$; Avantor, Gliwice, Poland). The absorbance of each dose was read using a spectrophotometer (Spectra Academy, $\mathrm{K}-\mathrm{MAC}$, Korea) at a wavelength of $570 \mathrm{~nm}$. The dye absorbance of the control cell was assumed as $100 \%$ and constituted a reference point in assessing the viability of the cells from the studied group.

The data obtained from the MTT assay was used to evaluate the potential interaction between the drugs. For this purpose, the Chou-Talalay median effect principle and CompuSyn software were applied. The method is based on the determination of combination indexes
(Cl) relative to the level of cytotoxicity. The analysis classifies relationships into three groups - synergistic $(\mathrm{Cl}<1)$, additive $(\mathrm{Cl}=1)$ and antagonistic $(\mathrm{Cl}>1)$ [9]. The results were presented as the $\mathrm{f}_{\mathrm{a}}-\mathrm{Cl}$ plot which was constructed by simulating $\mathrm{Cl}$ values over a range of fraction affected $\left(f_{a}\right)$ levels from 0.1 to 0.6 .

\section{Cell death analysis}

To analyse cell death double staining with the Apoptosis Assay Kit containing Annexin V Alexa Fluor 488 and Propidium lodide (Invitrogen; Thermo Fisher Scientific, Inc., Waltham, MA, USA) was performed according to instructions included by the manufacturer. The analysis was performed using a Guava 6HT-2L Cytometer (Merck $\mathrm{KGaA}$ ) and FlowJo vX 10.3 software.

\section{Western blot}

The semi-quantitative protein levels of vimentin, $\beta$-catenin, N-cadherin, E-cadherin, HIF-1 (Thermo Fisher Scientific), Bax (Abcam, Cambridge, United Kingdom) and Bcl-2 (Abcam) were performed using the standard protocol described in a previous study [10].

\section{Transwell migration assay}

To evaluate the migration potential of HepG2 cells transwell inserts (Corning, New York, USA) were used. Transwells were placed in a 24-well plate, where each well (the lower chamber) contained a $700 \mu \mathrm{M}$ EMEM medium with $15 \%$ FBS as a chemoattractant. After $24 \mathrm{~h}$ incubation, cells from the upper surface of the membrane were removed. The migration cells which adhered to the lower surface of the membrane were fixed in $3.6 \%$ PFA, incubated with $100 \%$ methanol and stained using $0.4 \%$ crystal violet. The preparations were examined using the Eclipse E800 microscope (Nikon) equipped with DS-5Mc-U1 CCD camera (Nikon) and NIS-Elements image analysis system (version 3.30; Nikon).

\section{Statistical analysis}

Statistical analysis was performed using GraphPad Prism version 6.0 (GraphPad Software, Inc., La Jolla, CA, USA). The results were considered statistically significant when $p<0.05$. For data obtained from MTT assay and Western blot analysis of HIF- $1 \alpha$, the Wilcoxon test was used where doses of DOX, DEX, and their combinations were compared to the hypothetical value for the control group absorbance considered as 100\%. In apoptosis analysis, the non-parametric Kruskal-Wallis with Dunn's post hoc test was used. In the case of the transwell migration assay and Western blot assay, 2way ANOVA with Dunnett's or Sidak's multiple comparisons test was performed. All data are presented by 


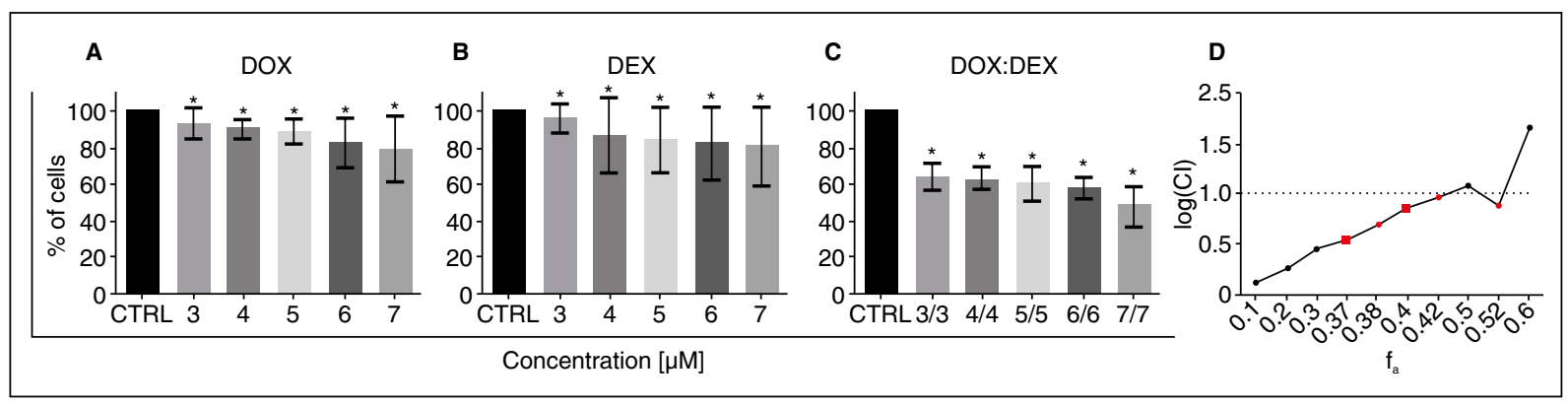

Figure 1. The cytotoxic effect of doxorubicin (DOX) and dexamethasone (DEX) individually and in combined treatment on cell viability of HepG2 cells. The cell viability analysis was based on the results obtained from the MTT assay. HepG2 cells were treated with DOX (A) and DEX (B) at concentrations from 3-7 $\mu \mathrm{M}$ for $24 \mathrm{~h}$ and their combination in ratio 1:1 (C). The data represent mean values \pm SD of 3 independent experiments $(n=3)$. Statistically significant differences were marked with '*' ( $p<0.05$; Wilcoxon test) (D). The combination index plot for DOX and DEX co-treatment in HepG2 cells in the range of $\mathrm{f}_{\mathrm{a}}$ from 0.1 to 0.6 . $\mathrm{Cl}<1$ - synergism, $\mathrm{Cl}=1$ - additive effect, $\mathrm{Cl}>1$ - antagonism. For real measuring points, the values have been marked in red

means \pm standard deviation (SD) of three independent experiments $(n=3)$.

\section{Results}

\section{The cytotoxic effect of DOX and DEX individually and in combined treatment on cells viability}

As shown in Figures $1 \mathrm{~A}$ and $1 \mathrm{~B}$, the use of $\mathrm{DOX}$ and DEX at doses from 3 to $7 \mu \mathrm{M}$ resulted in a dose-dependent decrease in cell viability. In the case of using DOX/DEX combination in the 1:1 ratio, it was also noted to decrease the population of live cells (Fig. 1C). All results obtained were statistically significant compared to the control (Fig.1A-C). The $\mathrm{IC}_{50}$ (half maximal inhibitory concentration; $f_{a}=0.5$ ) value for $24 \mathrm{~h}$ incubation was calculated in the CompuSyn program and reached 17.77 $\mu \mathrm{M}$ for DOX and 13.19 $\mu \mathrm{M}$ for DEX. Analysis of the type of interaction showed a CI value of $<1$, which was characteristic of synergism (Fig. 1D).

\section{Treatment of HepG2 cells with DOX, DEX and the combination of these drugs increased the population of apoptotic cells}

The analysis of cell death after treatment of HepG2 cells for 24 h with DOX, DEX, and their combination in a 1:1 ratio resulted in a decrease in the percentage of live cells (AV-/PI-) in comparison to untreated cells. In the case of $24 \mathrm{~h}$ incubation with $6 \mu \mathrm{M} \mathrm{DOX}, 6 \mu \mathrm{M}$ DEX and a combination of $6 \mu \mathrm{M}$ DOX with $6 \mu \mathrm{M}$ DEX the population of living cells also decreased. Except for the $3 \mu \mathrm{M}$ DEX, all results obtained were statistically significant (Fig. 2A). Figure 2A presented an increase in the percentage of apoptotic cells $(\mathrm{AV}+/ \mathrm{PI}-$ and $\mathrm{AV}+$ /
$\mathrm{PI}+$ ). All obtained results were statistically significant (Fig. 2A). The analysis also showed the necrotic population $(\mathrm{AV}-/ \mathrm{PI}+)$ (Fig. 2A). The representative plots were presented in Figure $2 \mathrm{~B}$.

The MTT analysis was also used to assess the effect of various doses of $\mathrm{CoCl}_{2}$ on the cell viability of the HepG2 line. The compound induces hypoxia in vitro by stabilizing HIF-1 $\alpha$. After $24 \mathrm{~h}$ incubation with $\mathrm{CoCl}_{2}$, the cell viability increased slightly but was statistically significant (Fig. 3A). Due to the HIF-1 $\alpha$ being the major regulator of response to hypoxia, the level of this protein was assessed using the Western blot method (Fig. 3B). The densitometric analysis showed that the highest level of HIF-1 $\alpha$ protein was recorded after using $80 \mu \mathrm{M} \mathrm{CoCl}_{2}$ (Fig. $3 \mathrm{C}$ ). Considering the obtained results during further experiments $80 \mu \mathrm{M}$ $\mathrm{CoCl}_{2}$ concentration was used to induce the hypoxia-mimic conditions.

\section{The effect of DOX and DEX individually and in combined treatment on migration potential of HepG2 cells}

The presented study mainly aimed to investigate the effect of DOX, DEX, and the combination of drugs in ratio $1: 1$ on the migration potential of HepG2 cells in both, normoxic and hypoxic conditions. For this purpose, recommended methods such as transwell migration assay and measurement of EMT markers with Western blot assay were used.

Figure 4A presents representative images of HepG2 cells with high migratory potential. For statistical analysis cells in normoxic and hypoxic conditions were counted on the underside of the transwell inserts. In the case of CTRL in normoxia, data was estimated as $100 \%$ and the results obtained for the drugs are presented 


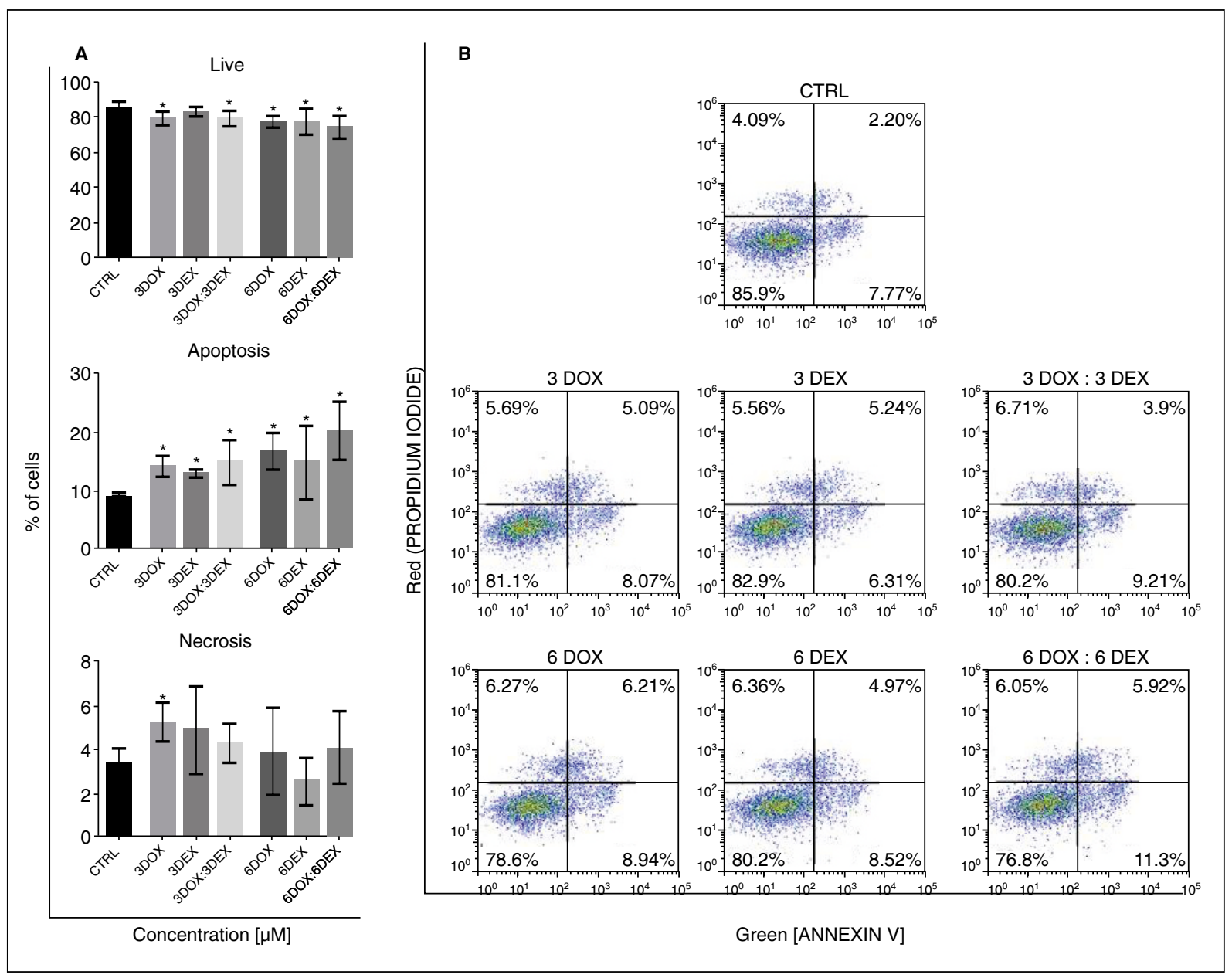

Figure 2. The effect of doxorubicin (DOX) and dexamethasone (DEX) individually and in combined treatment on cell death of HepG2 cells. The cytometric analysis of cell death using Annexin V Alexa Fluor 488 and PI double staining assay (A). The percentage of live, apoptotic, and necrotic cells. The HepG2 cells were treated with different concentrations of DOX and DEX ( $3 \mu \mathrm{M}$ or $6 \mu \mathrm{M})$ for $24 \mathrm{~h}$, and the combination of both drugs in ratio $1: 1$. ' $*$ ' indicate statistically significant differences in comparison to control cells $(P<0.05$; the non-parametric Kruskal-Wallis with Dunn's post hoc test) (B). The representative plots

as an average percentage in comparison to untreated cells (Fig. 4B). The obtained results suggest that the combination of $6 \mu \mathrm{M}$ DOX and DEX was more effective in reducing cell migration than $3 \mu \mathrm{M}$ DOX: $3 \mu \mathrm{M}$ DEX cotreatment in hypoxic conditions.

Data obtained in the Transwell migration assay was supported by Western blot analysis of selected EMT markers (Fig. 5). All results obtained in the analysis were evaluated in comparison to the HepG2 control cells without the $\mathrm{CoCl}_{2}$ pretreatment (estimated as 1). Assessment of vimentin level in HepG2 cells grown in normal conditions resulted in a decrease compared to CTRL. After treatment with DOX and DEX in ratio 1:1 increase was observed. Following incubation with $6 \mu \mathrm{M}$ DOX, a statistically significant increase in vimentin was noticed. In hypoxic condition, $3 \mu \mathrm{M} \mathrm{DOX}, 3 \mu \mathrm{M}$ DEX and the combination of drugs significantly decreased protein level in comparison to untreated HepG2 cells (Fig. 5A). Under both conditions, $6 \mu \mathrm{M}$ DEX alone and combined with $6 \mu \mathrm{M}$ DOX resulted in a significant decrease in the protein level in HepG2 (Fig. 5A).

Western blot evaluation of $\beta$-catenin showed that $3 \mu \mathrm{M}$ DOX and $6 \mu \mathrm{M}$ DEX promotes an increase in protein expression in comparison to untreated cells, respectively (Fig. 5B). The reductions in protein levels were observed for both combinations of drugs. All of the results were statistically significant. Moreover, a significant increase in $\beta$-catenin level for HepG2 with addition $\mathrm{CoCl}_{2}$ for CTRL, combined of $3 \mu \mathrm{M}$ DOX and DEX and $6 \mu \mathrm{M}$ DEX Fig. 5B) was noted.

Figure $5 \mathrm{C}$ presents the changes in the $\mathrm{N}$-cadherin level in normoxic and hypoxic conditions. Following treatment with $3 \mu \mathrm{M}$ DOX: $3 \mu \mathrm{M} \mathrm{DEX}$ and $6 \mu \mathrm{M} \mathrm{DOX}$ increase in $\mathrm{N}$-cadherin level was noticed. The untreated 


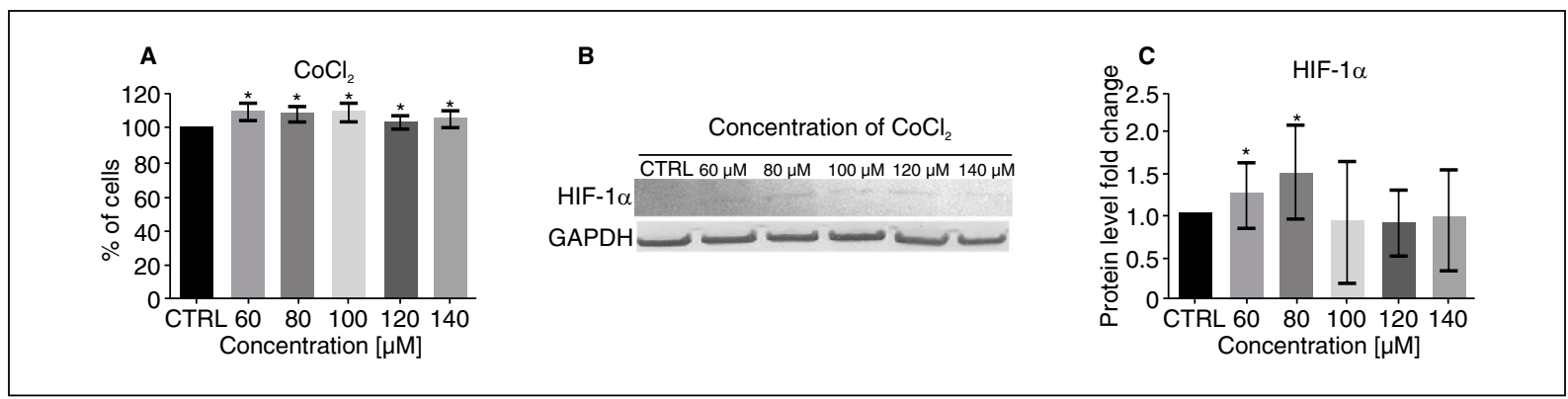

Figure 3. The effect of $\mathrm{CoCl}_{2}$ on the viability of HepG2 cells and the level of HIF-1 $\alpha$. (A) The cell viability analysis was based on the results obtained from the MTT assay. The HepG2 cells were treated with different concentrations of $\mathrm{CoCl}_{2}(60-140 \mu \mathrm{M})$ for $24 \mathrm{~h}$. The data represent mean values \pm SD of 3 independent experiments $(\mathrm{n}=3)$. Statistically significant differences are marked with ' ' ( $p<0.05$; Wilcoxon test); (B) Western blot analysis results of HIF-1 $\alpha$ and GAPDH in HepG2 cells following $\mathrm{CoCl}_{2}$ treatment for 24h; (C) Densitometric analysis of HIF-1 $\alpha$. Protein levels were normalized to untreated cells and the results were expressed as fold change vs. control (estimated as 1 ). Statistically significant differences are marked with '*' $(p<0.05$; Wilcoxon test). Data presented as mean \pm SD

HepG2 cells in hypoxic condition were characterized by an increase in protein expression in comparison to CTRL. Under both conditions, a statistically significant decrease in protein expression was observed for $3 \mu \mathrm{M}$ DOX, $3 \mu \mathrm{M}$ DEX $6 \mu \mathrm{M}$ DEX and $6 \mu \mathrm{M}$ combination of drugs (Fig. 5C).

The last of the analysed protein was E-cadherin. A statistically significant decrease was observed in the level of E-cadherin following treatment with $3 \mu \mathrm{M}$ DOX and $3 \mu \mathrm{M}$ DOX: $3 \mu \mathrm{M}$ DEX in comparison to untreated HepG2 cells in normoxia. In the same conditions, after $24 \mathrm{~h}$ incubation with $3 \mu \mathrm{M} \mathrm{DEX}$ and $6 \mu \mathrm{M} \mathrm{DEX} \mathrm{E-cadherin}$ levels increased (Fig. 5D). The addition of $\mathrm{CoCl}_{2}$ and $3 \mu \mathrm{M}$ DOX, $3 \mu \mathrm{M}$ DEX, $6 \mu \mathrm{M}$ DEX and combination of cytostatics at concentrations $3 \mu \mathrm{M}$ and $6 \mu \mathrm{M}$ resulted in a statistically significant increase in E-cadherin level in comparison to control in standard conditions.-The significant reductions in protein level in untreated HepG2 cells in hypoxic condition was noticed (Fig. 5D).

In the next step, Western blot analysis was applied to examine the expression of $\mathrm{Bax}$ and $\mathrm{Bcl}-2$, which are involved in apoptosis (Fig. 6A, B). As previously, evaluated HepG2 cells were treated with DOX, DEX, and a combination of drugs in ratio 1:1 in both, normoxia and hypoxia. Figure 6D presented that DOX at concentration $3 \mu \mathrm{M}$ and $6 \mu \mathrm{M}$ induced a statistically significant decrease in Bax/Bcl-2 ratio in comparison to CTRL in normal conditions Data obtained for HepG2 cells cultured with the addition of $\mathrm{CoCl}_{2}$ presented an increase in the $\mathrm{Bax} / \mathrm{Bcl}-2$ ratio compared to untreated cells in normoxia. Moreover, a statistically significant increase in $\mathrm{Bax} / \mathrm{Bcl}-2$ ratio for $24 \mathrm{~h}$ incubation with $6 \mu \mathrm{M} \mathrm{DOX}$, $6 \mu \mathrm{M}$ DEX and a combination of cytostatics were also noticed (Fig. 6D). These results may explain the inhibition of HepG2 migration potential after treatment in hypoxic conditions.

\section{Discussion}

Although many studies have been conducted on how to improve the TACE treatment strategy, the results are still not fully satisfying [11]. In the present study, it is suggested that the combination of a cytostatic commonly used during TACE — DOX with corticosteroid drug - DEX has the potential to improve TACE procedure outcome.

One of the problems arising during the TACE procedure is hypoxia. The cells under hypoxic conditions produce hypoxia-inducible angiogenic factors, e.g., VEGF, which stimulates the surrounding endothelial cells to form new blood vessels. Moreover, the lack of oxygen is one of the triggers for EMT [12]. This, in turn, is the basis for metastasis formation. As was shown by $\mathrm{Li}$ et al., the TACE procedure followed by a liver transplant results in a higher incidence of pulmonary metastasis in comparison to patients with liver transplants alone [13]. This implicates that poor response to the procedure may be connected with metastasis occurrence.

As indicated by Buschauer et al., HepG2 cells after DOX treatment undergo selection rather than death [14]. Moreover, the population of cells, which survived was characterized by an increased migratory potential and a decreased susceptibility to drug retreatment. In the case of this study, the authors applied $3 \mu \mathrm{M}$ and $6 \mu \mathrm{M}$ doses of DOX. Another drug applied in the study was DEX, which is a commonly used corticosteroid drug. However, some literature reports indicate its inhibiting effect on the migratory potential of cancer cells and blocking the hypoxia-induced EMT process [15]. Furthermore, the combination of DEX and DOX along with other cytostatics has been extensively tested for many types of cancer [16, 17]. As was indicated by Ogasawara et al., administration of DEX before TACE limits 


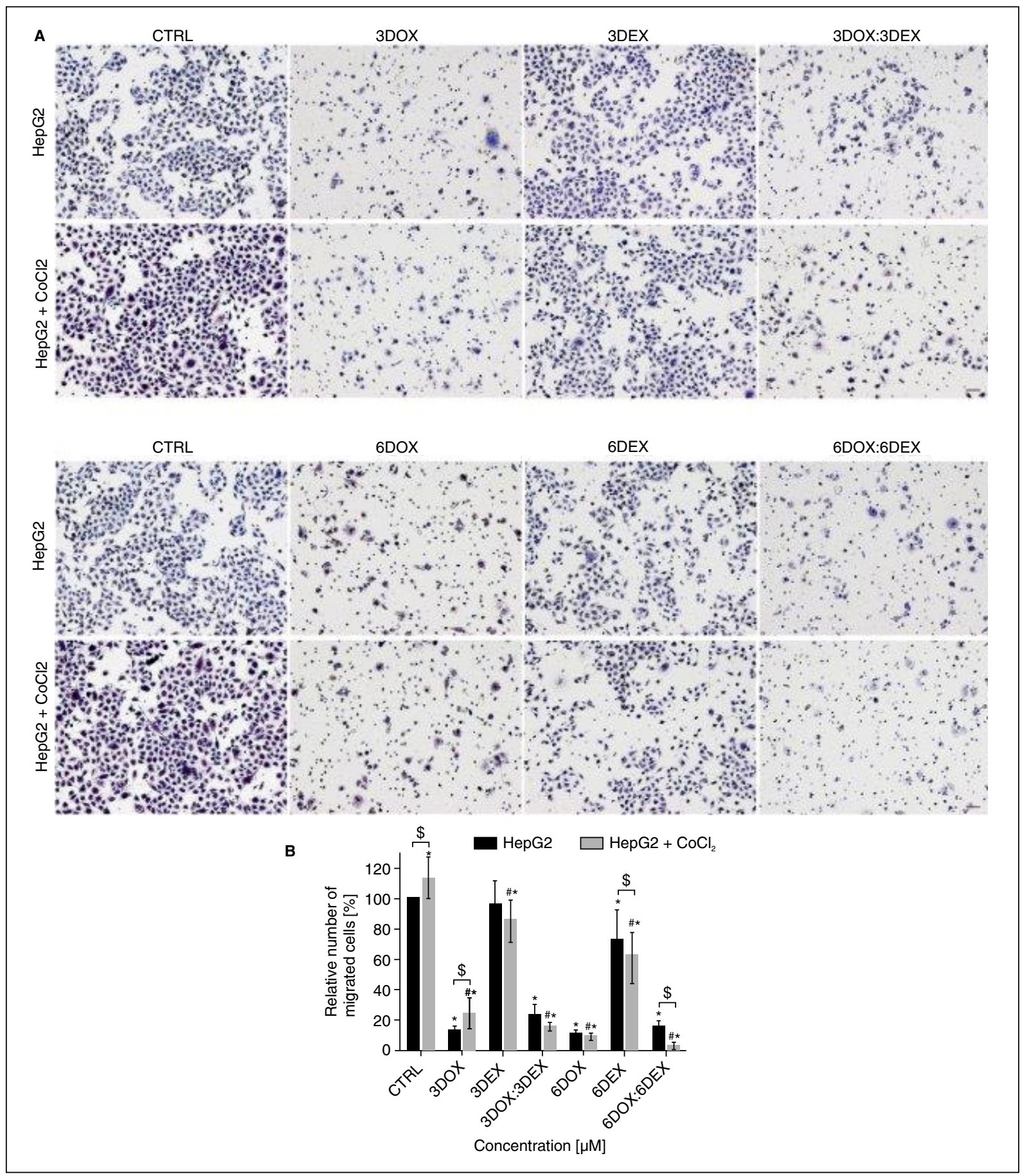

Figure 4. The effect of doxorubicin (DOX) and dexamethasone (DEX) individually and in combined treatment on migration potential of HepG2 cells - Transwell migration assay. HepG2 cells were treated with DOX, DEX, and the combination of drugs in ratio $1: 1$ in normoxia (HepG2) and hypoxia (HepG2 + $\mathrm{CoCl}_{2}$ ) for 24h; (A) The representative images of the results obtained in Transwell migration assay. Magnification 10x, Bar $=50 \mu \mathrm{m} ;(B)$ The average percentage of cells with high migratory potential relative to control cells in normoxia (estimated as $100 \%$ ). '*' indicate statistically significant differences in comparison to control cells in normoxia, '\#' to hypoxia, and '\$' between appropriate doses in normoxia and hypoxia $(P<0.05 ; 2$ way ANOVA with Dunnett's or Sidak's multiple comparisons test). Data presented as mean $\pm \mathrm{SD}$ 


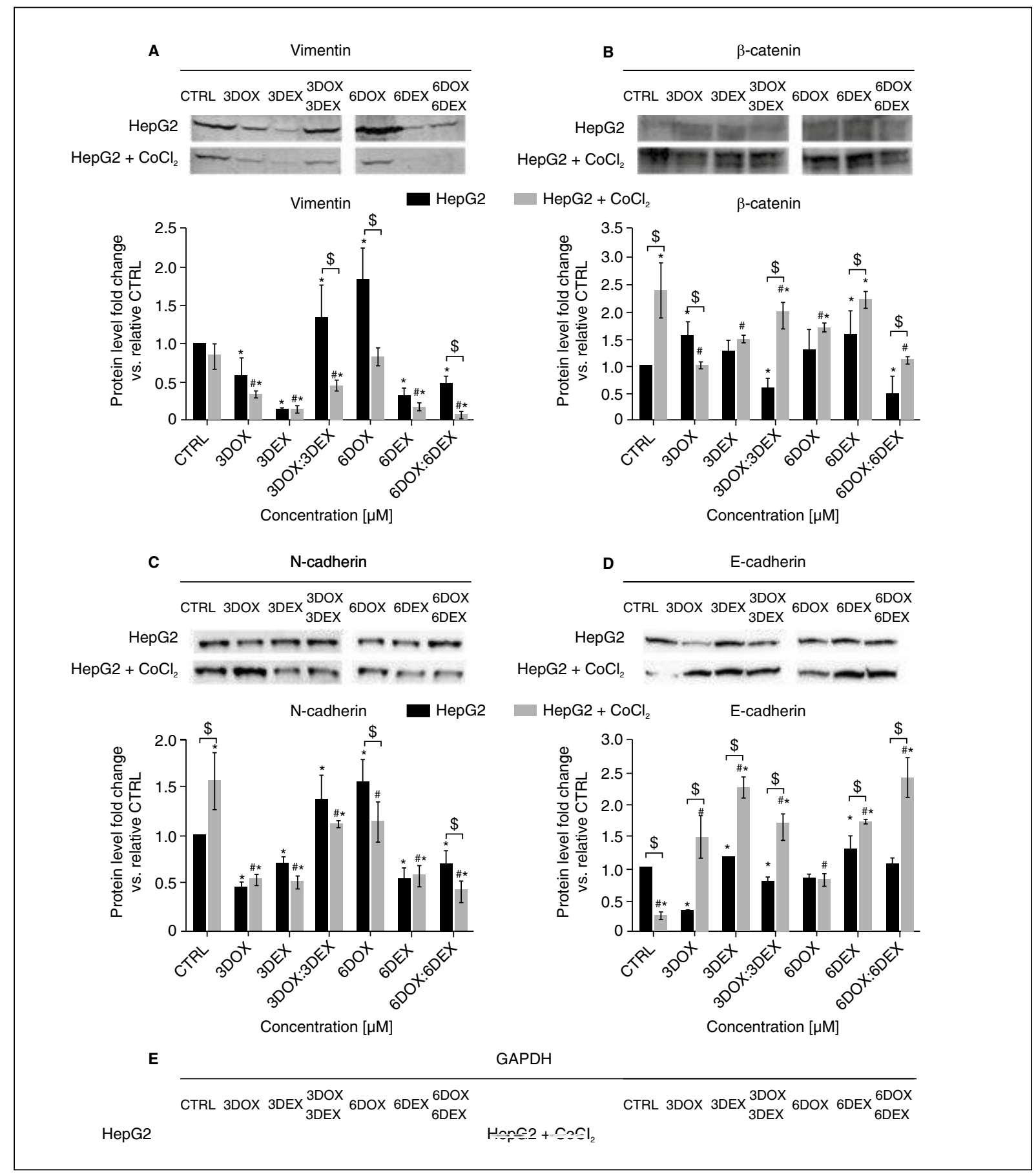

Figure 5. The effect of doxorubicin (DOX) and dexamethasone (DEX) individually and in combined treatment on migration potential of HepG2 cells - Western blot assay. HepG2 cells were treated with DOX, DEX, and the combination of drugs in ratio 1:1 in normoxia (HepG2) and hypoxia ( $\left.\mathrm{HepG} 2+\mathrm{CoCl}_{2}\right)$ for $24 \mathrm{~h}$. Representative pictures of membranes and graphs of (A) vimentin, (B) $\beta$-catenin, (C) N-cadherin, (D) E-cadherin, and (E) GAPDH are presented. Levels of the proteins were normalized to untreated cells and results were expressed as fold change vs. control in normoxia (estimated as 1). On the graphs ' $*$ ' indicate statistically significant differences for control cells in normoxia, '\#' in hypoxia, and '\$' between normoxic and hypoxic conditions ( $\mathrm{P}<0.05$; 2way ANOVA with Dunnett's or Sidak's multiple comparisons test). Data are presented as mean \pm SD 


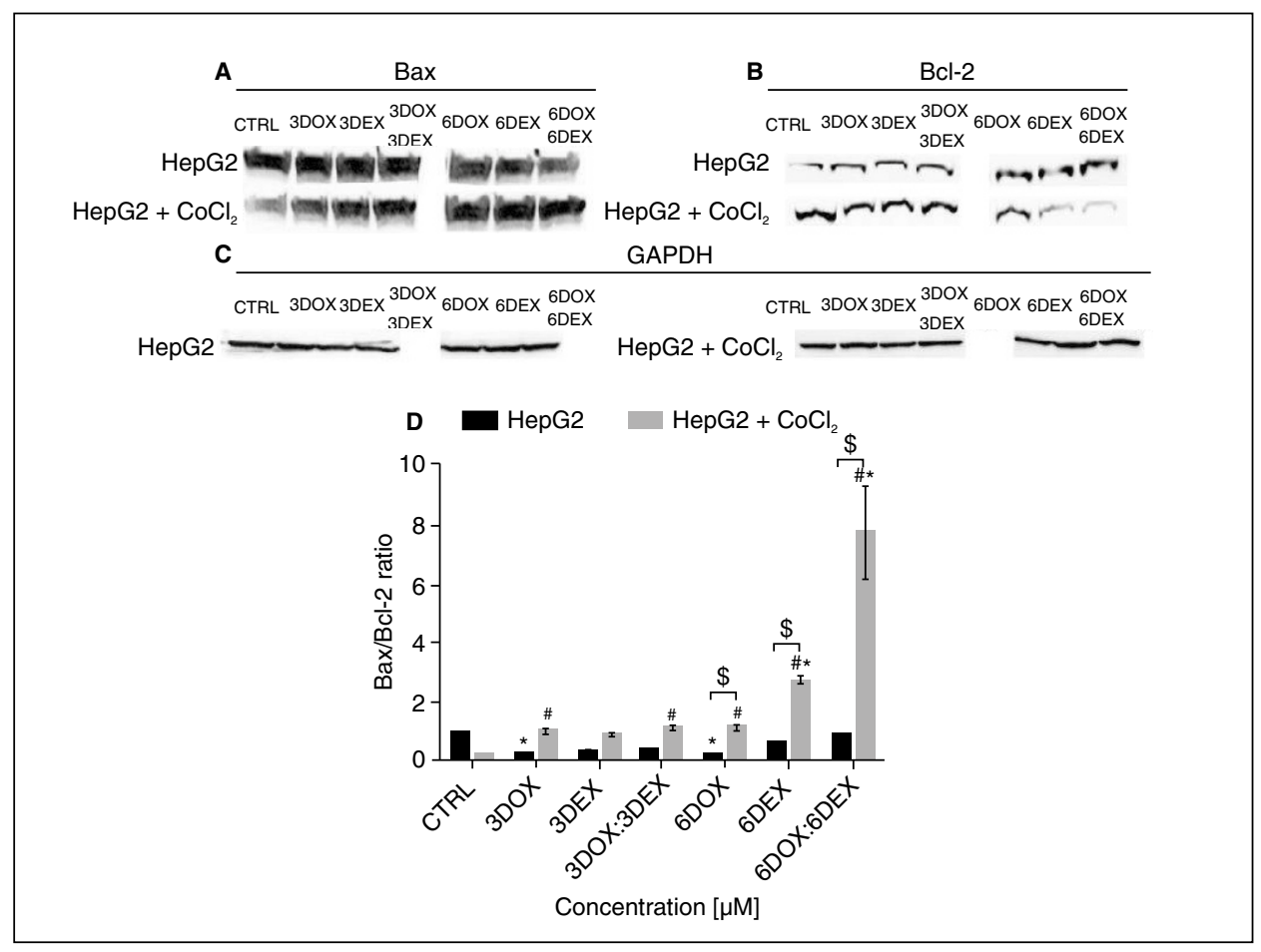

Figure 6. The effect of doxorubicin (DOX) and dexamethasone (DEX) individually and in combined treatment on the expression level of Bax and Bcl-2 in HepG2 cells - Western blot assay. HepG2 cells were treated with DOX, DEX, and the combination of drugs in ratio 1:1 in normoxia (HepG2) and hypoxia ( $\mathrm{HepG} 2+\mathrm{CoCl} 2)$ for $24 \mathrm{~h}$. Representative membranes of (A) Bax, (B) Bcl-2, (C) GAPDH and a graph of Bax/Bcl-2 ratio in HepG2 (D) are presented. Levels of the proteins were normalized to untreated cells and results were expressed as fold change vs. control in normoxia (estimated as 1). '*' indicate statistically significant differences for control cells in normoxia, '\#' in hypoxia, and '\$' between appropriate doses in normoxic and hypoxic conditions ( $P<0.05$; 2way ANOVA with Dunnett's or Sidak's multiple comparisons test). Data are presented as mean \pm SD

the incidences of fever, anorexia, and nausea/vomiting and also reduces recovery time [18].

In the case of this study, both DOX and DEX caused a dose-dependent decrease in the viability of HepG2 cells after $24 \mathrm{~h}$ treatment. However, the greatest reduction was visible in the case of the $1: 1$ combination of the drugs. The obtained value of $\mathrm{Cl}<1$ indicated synergistic interaction. Subsequent analyses confirmed a statistically significant increase in the percentage of apoptotic cells with a particular indication of a combination of cytostatics at a dose of $6 \mu \mathrm{M}$. This is consistent with the studies carried out by Dubbelboer et al. who showed that doxorubicin treatment results in a dose-dependent increase in the percentage of apoptotic cells [19]. The dose-effect disproportion between the studies may be connected with the phenomenon known as confluence dependent resistance, which involves a differentiated cell response to a drug depending on the confluence at the time of treatment. This is especially problematic considering the morphology of HepG2 cells, which tend to grow in clusters, making it difficult to assess the real confluence. To the authors' knowledge currently, there are no studies on the effect of DEX on liver cancer cells. However, apoptosis induction by the drug was noted in the case of colon cancer (LoVo) [20]. In turn, it did not show any significant effect on cell apoptosis in the case of non-small cell lung cancer (A549) cells, while for human ovarian cancer cells (HO-8910 and SKOV3) DEX treatment caused drug resistance to further chemotherapy [21, 22]. The diverse response of different cancer cell types to DEX treatment results in its frequent use in combination therapy rather than a single therapeutic agent. A combination with DOX has also been used for MCF-7 breast cancer cells [17]. However, the DOX: DEX combination in the context of liver cancer is innovative.

Numerous studies show that hypoxia promotes the migration and invasion of cancer cells [23]. The hypoxic conditions lead to the stabilization of HIF-1, which in turn causes an increase in the VEGF level [24]. VEGF promotes angiogenesis and EMT - both processes strictly connected to metastasis. Therefore, the main goal of the project was to assess the impact of the developed combination of drugs in the context of this 
process. To induce hypoxic condition, $\mathrm{CoCl}_{2}$ is often applied [25]. It mimics hypoxic conditions through the stabilization of HIF-1 protein. The present study also observed enhanced cell migration caused by hypoxic conditions. Transwell migration assay showed that pretreatment with $\mathrm{CoCl}_{2}$ resulted in intensified cell passage through the inserts pores. Moreover, in the case of DEX used in mono treatment enhanced cell migration was observed in comparison to the appropriate control, while for the combination of the drugs this effect was not visible. This is consistent with the observations of Guan et al. (2018) who noted increased migration of C6 glioma cells in response to DEX despite a reduction in cell proliferation [26]. In turn, Wu et al. (2019) indicate inhibition of HepG2 migration in response to DEX, however, both dose and incubation time were significantly greater [27]. The goal of the present research was to obtain the most efficient dose-effect ratio for the combination of the drugs. Selected doses of DOX and DEX, and in particular their combinations, led to a decrease in the number of cells with high migratory potential compared to the corresponding samples in normoxia.

The induction of hypoxia and the high migratory potential of cancer cells is closely related to EMT [28]. The main markers of this process are $\mathrm{N}$ - and $\mathrm{E}$-cadherin, vimentin, and $\beta$-catenin. For the cells pretreated with $\mathrm{CoCl}_{2}$, the level of E-cadherin is lower than for cells cultured in normoxic conditions, while the level of $\mathrm{N}$-cadherin is exactly the opposite. This suggests that also in the case of HepG2 cells, hypoxia-mimic conditions induced by $\mathrm{CoCl}_{2}$ result in an enhanced EMT process. This effect is limited by the developed combination of drugs, especially in the case of the 6 DOX: 6 DEX combination, which is manifested by the N/E-cadherin switch. Similarly, lower levels of vimentin expression after incubation with a combination of compounds were noted. It is commonly known that high levels of vimentin correlate with high cell mobility [29].

Hypoxia-induced migration may follow various pathways in cancer cells. Some studies point to the role of the $\mathrm{Wnt} / \beta$-catenin pathway. Moreover, this pathway is abnormally activated in the case of HCC. For this reason, it was also decided to assess the level of $\beta$-catenin. The authors suggest that elevated $\beta$-catenin levels in hypoxia may correlate with the activation of this pathway, which has also been shown in the study by Zhang et al. (2013) [30]. Moreover, the authors indicate the connection between the $\mathrm{Wnt} / \beta$-catenin and hypoxia-induced EMT, which is also visible in the present study. In turn, the developed combination inhibited this effect, which also translates into a decrease in Bcl-2 (antiapoptotic) and an increase in BAX (proapoptotic). Overexpression of Bcl-2 favours the EMT process through inhibition of E-cadherin. The obtained results show that the combination of DOX and
DEX used in hypoxic conditions may inhibit migration due to the promotion of apoptosis.

\section{Conclusions}

In summary, this study demonstrated that the developed innovative combination of DOX and DEX in the $1: 1$ ratio may limit hypoxia-induced enhancement in the migratory potential of HCC cells. The observed effect is connected with the inhibition of the EMT process and directing cells to death on the cellular level.

\section{Acknowledgements: This study was supported}

by a research task within the framework of the

Students Researches (Nicolaus Copernicus

University in Toruń, Faculty of Medicine, Collegium

Medicum in Bydgoszcz).

Competing interests: The authors declare that they have no competing interests.

\section{REFERENCES}

1. Galle PR, Tovoli F, Foerster F, et al. The treatment of intermediate stage tumours beyond TACE: From surgery to systemic therapy. J Hepatol. 2017; 67(1): 173-183, doi: 10.1016/j.jhep.2017.03.007, indexed in Pubmed: 28323121

2. El Fouly A, Ertle J, El Dorry A, et al. In intermediate stage hepatocellular carcinoma: radioembolization with yttrium 90 or chemoembolization? Liver Int. 2015; 35(2): 627-635, doi: 10.1111/liv.12637, indexed in Pubmed: 25040497.

3. Boulin M, Delhom E, Pierredon-Foulongne MA, et al. Transarterial chemoembolization for hepatocellular carcinoma: An old method, now flavor of the day. Diagn Interv Imaging. 2015; 96(6): 607-615, doi: 10.1016/j.diii.2015.04.005, indexed in Pubmed: 25998996.

4. Asghar U, Meyer T. Are there opportunities for chemotherapy in the treatment of hepatocellular cancer? J Hepatol. 2012; 56(3): 686-695, doi: 10.1016/j.jhep.2011.07.031, indexed in Pubmed: 21971559.

5. Lau CK, Yang ZF, Ho DW, et al. An Akt/hypoxia-inducible factor-1alpha/platelet-derived growth factor-BB autocrine loop mediates hypoxia-induced chemoresistance in liver cancer cells and tumorigenic hepatic progenitor cells. Clin Cancer Res. 2009; 15(10): 3462-3471, doi: 10.1158/1078-0432.CCR-08-2127, indexed in Pubmed: 19447872.

6. De Francesco EM, Maggiolini M, Musti AM. Crosstalk between Notch, HIF-1 $\alpha$ and GPER in Breast Cancer EMT. Int J Mol Sci. 2018; 19(7), doi: 10.3390/ijms 19072011, indexed in Pubmed: 29996493.

7. Roche J. Erratum: Roche, J. The Epithelial-to-Mesenchymal Transition in Cancer. Cancers, 2018, 10, 52. Cancers (Basel). 2018; 10(3), doi: 10.3390/cancers10030079, indexed in Pubmed: 29562660

8. Simko V, Takacova M, Debreova M, et al. Dexamethasone downregulates expression of carbonic anhydrase IX via HIF- $1 \alpha$ and NF- $\kappa$ B-dependent mechanisms. Int J Oncol. 2016; 49(4): 1277-1288, doi: 10.3892/ijo.2016.3621, indexed in Pubmed: 27431580

9. Chou TC. Theoretical basis, experimental design, and computerized simulation of synergism and antagonism in drug combination studies. Pharmacol Rev. 2006; 58(3): 621-681, doi: 10.1124/pr.58.3.10, indexed in Pubmed: 16968952

10. Krajewski A, Gagat M, Żuryń A, et al. Cyclin F is involved in response to cisplatin treatment in melanoma cell lines. Oncol Rep. 2020; 43(3): 765-772, doi: 10.3892/or.2020.7465, indexed in Pubmed: 32020229.

11. Lencioni R, Petruzzi P, Crocetti L. Chemoembolization of hepatocellular carcinoma. Semin Intervent Radiol. 2013; 30(1): 3-11, doi: 10.1055/s0033-1333648, indexed in Pubmed: 24436512.

12. Choi BJ, Park SA, Lee SY, et al. Hypoxia induces epithelial-mesenchymal transition in colorectal cancer cells through ubiquitin-specific protease 47-mediated stabilization of Snail: A potential role of Sox9. 
Sci Rep. 2017; 7(1): 15918, doi: 10.1038/s41598-017-15139-5, indexed in Pubmed: 29162839.

13. Li HL, Ji WB, Zhao R, et al. Poor prognosis for hepatocellular carcinoma with transarterial chemoembolization pre-transplantation: retrospective analysis. World J Gastroenterol. 2015; 21(12): 3599-3606, doi: 10.3748/wjg.v21.i12.3599, indexed in Pubmed: 25834326

14. Buschauer S, Koch A, Wiggermann P, et al. Hepatocellular carcinoma cells surviving doxorubicin treatment exhibit increased migratory potential and resistance to doxorubicin re-treatment. Oncol Lett. 2018; 15(4): 4635-4640, doi: 10.3892/ol.2018.7887, indexed in Pubmed: 29541235.

15. Kim JHo, Hwang YJ, Han $\mathrm{SH}$, et al. Dexamethasone inhibits hypoxia-induced epithelial-mesenchymal transition in colon cancer. World J Gastroenterol. 2015; 21(34): 9887-9899, doi: 10.3748/wjg.v21. i34.9887, indexed in Pubmed: 26379394

16. Hassoun $\mathrm{H}$, Reich $\mathrm{L}$, Klimek VM, et al. Doxorubicin and dexamethasone followed by thalidomide and dexamethasone is an effective well tolerated initial therapy for multiple myeloma. $\mathrm{Br} J$ Haematol. 2006; 132(2): 155-161, doi: 10.1111/j.1365-2141.2005.05848.x, indexed in Pubmed: 16398649

17. Chaikomon K, Chattong S, Chaiya T, et al. Doxorubicin-conjugated dexamethasone induced MCF-7 apoptosis without entering the nucleus and able to overcome MDR-1-induced resistance. Drug Des Devel Ther. 2018; 12: 2361-2369, doi: 10.2147/DDDT.S168588, indexed in Pubmed: 30122894.

18. Ogasawara S, Chiba T, Ooka Y, et al. A randomized placebo-controlled trial of prophylactic dexamethasone for transcatheter arterial chemoembolization. Hepatology. 2018; 67(2): 575-585, doi: 10.1002/hep.29403, indexed in Pubmed: 28746788.

19. Dubbelboer IR, Pavlovic N, Heindryckx F, et al. Liver cancer cell lines treated with doxorubicin under normoxia and hypoxia: cell viability and oncologic protein profile. Cancers (Basel). 2019; 11(7), doi: 10.3390/cancers11071024, indexed in Pubmed: 31330834.

20. He J, Zhou J, Yang W, et al. Dexamethasone affects cell growth/apoptosis/chemosensitivity of colon cancer via glucocorticoid receptor $\alpha / \mathrm{NF}-\kappa$ B. Oncotarget. 2017; 8(40): 67670-67683, doi: 10.18632/oncotarget.18802, indexed in Pubmed: 28978062

21. Chen $Y X$, Wang Y, Fu CC, et al. Dexamethasone enhances cell resistance to chemotherapy by increasing adhesion to extracellular matrix in human ovarian cancer cells. Endocr Relat Cancer. 2010; 17(1): 39-50, doi: 10.1677/ERC-08-0296, indexed in Pubmed: 19776289.

22. Wang LJ, Li J, Hao FR, et al. Dexamethasone suppresses the growth of human non-small cell lung cancer via inducing estrogen sulfotransferase and inactivating estrogen. Acta Pharmacol Sin. 2016; 37(6): 845-856, doi: 10.1038/aps.2016.39, indexed in Pubmed: 27133297.

23. Ou XW, Wang RX, Kang MF, et al. Hypoxia promotes migration and invasion of gastric cancer cells by activating HIF- $1 \alpha$ and inhibiting NDRG2 associated signaling pathway. Eur Rev Med Pharmacol Sci. 2018; 22(23): 8237-8247, doi: 10.26355/eurrev_201812_16518, indexed in Pubmed: 30556863.

24. Lee JW, Ko J, Ju C, et al. Hypoxia signaling in human diseases and therapeutic targets. Exp Mol Med. 2019; 51(6): 1-13, doi: 10.1038/s12276019-0235-1, indexed in Pubmed: 31221962.

25. Muñoz-Sánchez J, Chánez-Cárdenas ME, Orozco-lbarra M et al. Aged garlic extract and S-allylcysteine prevent apoptotic cell death in a chemical hypoxia model. Biol Res. 2016; 49(4): 7-570, doi: 10.1186/s40659-016-0067-6, indexed in Pubmed: 26830333.

26. Guan Y, Chen J, Zhan Y, et al. Effects of dexamethasone on C6 cell proliferation, migration and invasion through the upregulation of AQP1. Oncol Lett. 2018; 15(5): 7595-7602, doi: 10.3892/ol.2018.8269, indexed in Pubmed: 29740485

27. Wu $\mathrm{Y}, \mathrm{Xia} \mathrm{R}$, Dai $\mathrm{C}$, et al. Dexamethasone inhibits the proliferation of tumor cells. Cancer Manag Res. 2019; 11: 1141-1154, doi: 10.2147/CMAR.S187659, indexed in Pubmed: 30774442.

28. Zhang XD, Dong XQ, Xu JL, et al. Hypoxia promotes epithelial-mesenchymal transition of hepatocellular carcinoma cells via inducing Twist1 expression. Eur Rev Med Pharmacol Sci. 2017; 21(13): 3061-3068, indexed in Pubmed: 28742200

29. Strouhalova K, Přechová M, Gandalovičová A, et al. Vimentin Intermediate Filaments as Potential Target for Cancer Treatment. Cancers (Basel). 2020; 12(1), doi: 10.3390/cancers12010184, indexed in Pubmed: 31940801

30. Zhang Qi, Bai $X$, Chen $W$, et al. Wnt $/ \beta$-catenin signaling enhances hypoxia-induced epithelial-mesenchymal transition in hepatocellular carcinoma via crosstalk with hif- $1 \alpha$ signaling. Carcinogenesis. 2013 34(5): 962-973, doi: 10.1093/carcin/bgt027, indexed in Pubmed: 23358852. 